\title{
CCD IMAGING OF NGC 4861: IONIZED GAS PROPERTIES AND STELLAR FORMATION
}

\author{
ClarisSA S. BARTH ${ }^{1}$ \\ Departmento de Astronomia, Instituto de Física-UFRGS, CP 15051, Porto Alegre, 91500, RS, Brasil \\ Electronic mail: clarissa@astro.umontreal.ca \\ JoRd CEPA AND JosÉ M. VILCHEZ \\ Instituto de Astrofísica de Canarias, E-38200 La Laguna, Tenerife, Spain \\ Electronic mail: jcn@iac.es, jvm@iac.es \\ HORACIO A. DOTTORI \\ Departments de Astronomia, Instituto de Física-UFRGS, CP 15051, Porto Alegre, 91500, RS, Brasil \\ Electronic mail: dottori@if1.ufrgs.br \\ Received 1993 February 9; revised 1994 May 20
}

\begin{abstract}
This work undertakes the study of the galaxy NGC 4861, using imaging centered at the strong lines $\mathrm{H} \alpha, \mathrm{H} \beta$, [O III] $\lambda 5007$ and nearby continua. Integrated, absolute fluxes of $28 \mathrm{H}$ II regions, and the region known as the bright knot, have been measured showing correlations between the equivalent width of $\mathrm{H} \beta$ and the excitation index $\log ([\mathrm{O} \mathrm{III}] / \mathrm{H} \beta)$. An excitation gradient has been detected along the chain of $\mathrm{H}$ II regions extending northwards from the bright knot, which is unusual for irregular galaxies like NGC 4861. We suggest that this gradient is due to different stages of evolution, or ages, of the $\mathrm{H}$ II regions. The continuum fluxes of eight emissionless associations and the continuum colors of the companion galaxy IC 3961 have also been measured, and the presence of ionized gas cannot be detected. The age and mass of the stellar population and ionizing stars of the bright knot were subsequently estimated. It was also verified that this region is the most important burst of star formation in NGC 4861.
\end{abstract}

\section{INTRODUCTION}

From the theoretical point of view, it is possible to derive the physical conditions in emission nebulae, thanks to the observation of appropriate emission lines. However, this approach generally ends in failure due to the difficulty of observing temperature indicators which involve very weak lines such as [O III] $\lambda 4363 \AA$. The determination of the heavy elements' content is of great importance to address problems such as the formation of galaxies. Searle (1971) detected a gradient in the excitation index $\log ([\mathrm{O} \mathrm{III}] / \mathrm{H} \beta)$ of $\mathrm{H}$ II regions in the disk of spiral galaxies, which was explained as produced by a difference in the content of heavy elements among the $\mathrm{H}$ II regions. More recent works (Pagel et al. 1979; McCall et al. 1985, hereafter referred to as MRS) have shown that a better metallicity indicator is $([\mathrm{O} \mathrm{II}]+[\mathrm{O} \mathrm{III}]) /$ $\mathrm{H} \beta$. At any rate, the $[\mathrm{O} \mathrm{III}] / \mathrm{H} \beta$ ratio can be used as a metallicity indicator, taking into account that there is an empirically determined (MRS) dependence between the intensities of the $[\mathrm{O}$ III $] \lambda 5007$ and [O II $] \lambda 3727$ lines.

The observation of intense lines also makes possible the determination of other properties, like the internal absorption of the parent galaxy through the Balmer decrement, considering that the absorption due to our galaxy in the direction of NGC 4861 is evaluated at $0.2 \mathrm{mag}$ in $V$ (de Vaucouleurs et al. 1976). Moreover, when line observations are complemented with continuum ones, we can obtain information

${ }^{1}$ Brazilian CNPq fellow. about the optical properties of the ionizing association of the emitting nebulae.

In this paper we study NGC 4861, a peculiar galaxy at the distance of $17.7 \mathrm{Mpc}$, given a systemic velocity of 885 $\mathrm{km} \mathrm{s}^{-1}$ (Fisher \& Tully 1981), and an $H_{0}$ of 50 $\mathrm{km} \mathrm{s}^{-1} \mathrm{Mpc}^{-1}$. In the Arp Atlas of Peculiar Galaxies (ARP266, Arp 1966) it is described as "an object with irregular clumps, resolved into knots with a very bright knot (diameter $=1 \mathrm{kpc}$ ) at the southeastern end." It has the shape of an elongated streak or edge-on disk (Dinnerstein \& Shields 1986). The bright knot (hereafter BK) is the most striking feature of NGC 4861, at the end of a chain of bright spots towards the north direction, superimposed on an elliptical, older population distribution which we identify as the NGC 4861's companion galaxy IC 3961 (Zwicky 1961). These features, which cover a $5 \times 2 \mathrm{kpc}$ area, strongly resemble the ensemble 30 Dor nebula plus Bar in the LMC, though the $\mathrm{BK}$ is much larger in size and more luminous than 30 Dor. The knots are in fact $\mathrm{H}$ II regions (Hodge 1983) chained in a tail-like structure connected to the BK. They are more or less aligned with the major axis of IC 3961. The BK of the NGC 4861 was observed spectroscopically by Dinnerstein \& Shields (1986), who find an oxygen abundance of $12+\log (\mathrm{O} / \mathrm{H})=8.1$ and whose spectrum clearly reveals the presence of WR features typical of WN and WC types, at $\lambda \lambda 4650$ and $5800 \AA$.

In order to study globally the physical conditions of the emitting gas, the characteristics of the ionizing associations and other detectable stellar associations, as well as those of the underlying older stellar population, in NGC 4861 and the 
TABLE 1. Log of observations: first column: date of observation; 2nd column: object; 3rd column: observed filters and number of observations; column 4: exposure time per individual image.

\begin{tabular}{lllc}
\hline \hline DATE & OBJECT & FILTER & EXP. TIME (s) \\
\hline $28 \mathrm{Feb}$ & $\mathrm{HD} 84937$ & $\mathrm{H} \alpha(2), \mathrm{H} \beta(2),[\mathrm{OIII}](2)$ & 20 \\
& & $\mathrm{CH} \alpha(2), \mathrm{CH} \beta(2), \mathrm{C}[\mathrm{OIII}]$ & 6 \\
\hline 2 Mar & $\mathrm{HD} 84937$ & $\mathrm{H} \alpha(2), \mathrm{H} \beta(2),[\mathrm{OIII}](2)$ & 20 \\
& & $\mathrm{CH} \alpha(2), \mathrm{CH} \beta(2), \mathrm{C}[\mathrm{OIII}](2)$ & 6 \\
\hline $5 \mathrm{Mar}$ & $\mathrm{HD} 84937$ & $\mathrm{H} \alpha(2), \mathrm{H} \beta(2),[\mathrm{OIII}](2)$ & 20 \\
& & $\mathrm{CH} \alpha(2), \mathrm{CH} \beta(2), \mathrm{C}[\mathrm{OIII}](2)$ & 6 \\
& \multirow{N}{*}{$\mathrm{HC} 4861$} & $\mathrm{H} \alpha(2), \mathrm{CH} \alpha(3)$ & 1800 \\
& & $\mathrm{H} \beta(3), \mathrm{CH} \beta(1)$ & \\
\hline 6 Mar & $\mathrm{HD} 84937$ & $\mathrm{H} \alpha(2), \mathrm{H} \beta(2),[\mathrm{OIII}](2)$ & 6 \\
& & $\mathrm{CH} \alpha(2), \mathrm{CH} \beta(2), \mathrm{C}[\mathrm{OIII}](2)$ & 1800 \\
& \multirow{N}{*}{$\mathrm{HGC} \mathrm{4861}$} & $\mathrm{H} \alpha(3), \mathrm{CH} \alpha(2)$ & \\
& & $\mathrm{H} \beta(2), \mathrm{CH} \beta(3)$ & \\
& {$[\mathrm{OIII}](2), \mathrm{C}[\mathrm{OIII}](3)$} & \\
\hline \hline
\end{tabular}

companion galaxy IC 3961, we undertook a program of CCD imaging, with narrowband interference filters centered at $\mathrm{H} \alpha$, $\mathrm{H} \beta$, [O III], and continua at 4556, 5290, and $6300 \AA$. We used the Jacobus Kapteyn $1.0 \mathrm{~m}$ Telescope, at the Roque de los Muchachos Observatory, in La Palma.

\section{THE OBSERVATIONS}

The data were collected during a seven night run in February-March, 1989 (see Table 1), with a CCD camera attached to the Cassegrain $f / 15$ focus of the $1.0 \mathrm{~m}$ Jacobus Kapteyn Telescope, in the Roque de los Muchachos Observatory, La Palma. The detector used was a GEC CCD of $400 \times 590$ pixels of $22 \mu \mathrm{m}$ each, giving a scale of 0.30 arcsec per pixel. Typical readout noise was 9 e pix $^{-1}$.

We used narrowband interference filters centered on the redshifted emission lines $\mathrm{H} \beta$, [O III] $\lambda 5007$, and $\mathrm{H} \alpha$, as well as on the continua at 4556,5290 , and $6300 \AA$ (see Table 2). Throughout the run, the seeing ranged from good to very good, varying between 0.8 and 1.2 arcsec, and exceptionally reaching 1.5 arcsec. Each image was taken with an integration time of $1800 \mathrm{~s}$. Four frames or more were coadded for each filter.

Spectrophotometric standard stars from Oke \& Gunn (1983) were observed to calibrate the observations in absolute flux. For the atmospheric extinction we adopted the Roque de los Muchachos Observatory's standard curve.

\section{DATA REDUCTION}

The data were reduced using the software package IRAF V.2.8, installed on a Sun Sparc 1+ Workstation. Bias frames

TABLE 2. Filter parameters. $T_{l}$ is the filter transmission at the wavelength of the line.

\begin{tabular}{cccc}
\hline \hline FILTER & $\lambda_{\circ}$ & FWHM & $\mathrm{T}_{l}$ \\
\hline $\mathrm{H} \alpha$ & 6563 & $57.1 \AA$ & 0.45 \\
$\mathrm{H} \beta$ & 4866 & $51.7 \AA$ & 0.48 \\
{$[\mathrm{OIII}]$} & 5012 & $50.0 \AA$ & 0.54 \\
$\mathrm{CH} \alpha$ & 6300 & $132.0 \AA$ & - \\
$\mathrm{CH} \beta$ & 4556 & $114.0 \AA$ & - \\
$\mathrm{C}[\mathrm{OIII}]$ & 5290 & $113.0 \AA$ & - \\
\hline \hline
\end{tabular}

were taken every night and averaged to give a master bias in order to take into account pixel-to-pixel variations. As the master bias presented a very constant value along its pixel distribution, we were able to subtract its mean value from the images, and use the overscan of each frame to obtain the bias level. Also, twilight flatfields of blank fields (Christian et al. 1985) were taken, for each filter, every night. After bias subtraction, the flatfields were averaged and normalized for every night in a given filter and the debiased images were divided by their corresponding flatfields. The sky level was determined and subtracted from each image as the mean of a region of the frame clear of objects. Limiting flux logarithms were -17.30 and -18.70 for $\mathrm{H} \alpha$ and its continuum, -17.10 and -18.50 for $\mathrm{H} \beta$ and its continuum, and -17.30 and -18.60 for [O III] and its continuum; in $\mathrm{erg} \mathrm{cm}^{-2} \mathrm{~s}^{-1} \mathrm{pix}^{-1}$ (and $\AA^{-1}$ for the continuum data).

\subsection{Absolute Flux Calibration}

We define $n_{g \lambda}$ and $n_{* \lambda}$ as the number of integrated counts of the object outside the atmosphere, per second, in a wavelength $\lambda$, and of the calibration star, respectively. Line centered filters receive continuum photons in addition to those of the emission lines. Thus, in order to disentangle the line fluxes, we used the following definitions and algorithm, assuming a top-hat spectral response and a flat continuum for the object at the wavelength considered:

$K_{\lambda}=$ response of the equipment's optics, including the detector, at $\lambda$.

$\Delta_{\lambda}=$ the filter's equivalent width.

$T_{l}=$ filter transmission on the line.

$I_{l}=$ number of line photons.

$F_{c}=$ number of continuum photons per unit $\lambda$.

For a galaxy, with the filter centered on the line 1 , the photon counts are given by

$$
n_{g l}=K_{l}\left(\Delta_{l} F_{g c}+T_{l} I_{g l}\right) \text {, }
$$

and for a filter centered in the continuum they are given by

$$
n_{g c}=K_{c} \Delta_{c} F_{g c} \text {. }
$$

As comparison stars do not present emission lines, the corresponding Eqs. (1) and (2) are

$$
\begin{aligned}
& n_{* l}=K_{l} \Delta_{l} F_{* l}, \\
& n_{* c}=K_{c} \Delta_{c} F_{* c} .
\end{aligned}
$$

The values $F_{* l}$ and $F_{* c}$ are obtained from Oke \& Gunn (1983), and the convolution of the filters parameters with the optics is obtained from

$$
\begin{gathered}
K_{l} \Delta_{l}=\frac{n_{* l}}{F_{* l}}, \\
K_{c} \Delta_{c}=\frac{n_{* c}}{F_{* c}} .
\end{gathered}
$$

For the galaxy we define

$$
\eta_{g l}=\frac{n_{g l}}{K_{l} \Delta_{l}}=F_{g c}+\frac{T_{l}}{\Delta_{l}} I_{g l}
$$

and, likewise, 
$\eta_{g c}=F_{g c}$.

As the filter transmission is known, substituting (8) into (7), we have

$I_{g l}=\left(\eta_{g l}-\eta_{g c}\right) \frac{\Delta_{l}}{T_{l}}$.

Balmer emission line intensities were corrected considering the effect of the underlying absorption line, produced mainly by $\mathrm{B}, \mathrm{A}$, and $\mathrm{F}$ stars from the ionizing association. For the galaxy, this correction has been based on the average value calculated by MRS, which is $1.9 \AA$ for the absorption's equivalent width. The true emission intensities were estimated taking into account the ratio between the equivalent widths of the line filter and the underlying absorption. For $\mathrm{H} \alpha$ this value is approximately $3.3 \%$, and for $\mathrm{H} \beta 3.8 \%$. The influence of the absorption lines of the standard stars was corrected by using the spectral energy distribution of Oke \& Gunn (1983). These values are $7 \%$ for $\mathrm{H} \beta$, and $3.2 \%$ for $\mathrm{H} \alpha$.

The continua at $\mathrm{H} \beta$ and [O III] $\lambda 5007$ were obtained from the measured ones at 4556,5290 , and $6300 \AA$, by a linear interpolation:

$$
\begin{aligned}
& \eta_{g \mathrm{H} \beta c}=\eta_{\lambda 4556} \frac{5290-4861}{5290-4556}+\eta_{\lambda 5290} \frac{4861-4556}{5290-4556}, \\
& \eta_{g[\mathrm{O} \mathrm{III}] c}=\eta_{\lambda 4556} \frac{5290-5007}{5290-4556}+\eta_{\lambda 5290} \frac{5007-4556}{5290-4556} .
\end{aligned}
$$

The intensity of [O III] $\lambda 5007$ was multiplied by 1.33 to obtain the total value of [O III] $\lambda(4959,5007)$. For $\mathrm{H} \alpha$ we adopted $\eta_{g \mathrm{H} \alpha c}=\eta_{g 6300}$.

We can take into account the contribution of $[\mathrm{O} \mathrm{I}] \lambda 6300$ and [S III] $\lambda 6312$ to the $H \alpha c$ flux we measured, with the line fluxes published by Dinerstein \& Shields (1986). For the BK, the $[\mathrm{O}$ I] $\lambda 6300$ line flux represents $2 \%$ of the total flux in the $\mathrm{H} \alpha c$ filter, and for [S III] $\lambda 6312,2.2 \%$.

[N II] $\lambda 6584$ has an intensity of $1 \%$ in $\mathrm{H} \alpha$, and the filter transmission at $6584 \AA$ is about $6 \%$, causing a "contamination" in $\mathrm{H} \alpha$ of less than $0.06 \%$.

\subsection{External Errors}

In what follows we discuss the sources of error that may be present in our work. The Poissonian noise $\sqrt{N} / N$ for the integrated counts is about $1 \%$ in $\mathrm{H} \alpha$, in an $\mathrm{H}$ II region. For the $\mathrm{BK}$, where the counts are higher, the noise is smaller than $0.3 \%$. The presence of strong WR features in the BK (Dinnerstein \& Shields 1986) may introduce an anomalous continuum for [O III] and/or $\mathrm{H} \beta$, and could also affect the measurements of the Balmer line fluxes and $W(\mathbf{H} \beta)$. In principle, we believe that the effect is not very strong in our data since our measured $W(\mathbf{H} \beta)$ is consistent with that of Dinnerstein \& Shields (1986).

The atmospheric extinction correction applied to the images is one of the main sources of error. The long integration times imply varying airmasses along the image exposure, which is not considered in the data reduction process, for which we adopt an average value for the airmass.
Filter parameters are also sources of errors. The differences between the environmental temperature during the observations and that prevailing in the laboratory [at which the filter's transmission curve is measured $\left(20^{\circ} \mathrm{C}\right)$ ], cause a shift of the maximum of the transmission curve, which must be taken into account in the filters used to measure line intensities. This effect has been considered carefully in our reductions, since we have registered the instrumental temperature along the observations. The cosmological redshift of the emission lines of the galaxy was also taken into account when determining $T_{l}$.

The statistical fluctuation of the counts in different frames also introduces an error. For example, five independent observations of the standard star give a mean of 23000 counts with a standard deviation of $4.5 \%$, after the correction for atmospheric extinction (with airmasses between 1.03 and 1.65).

A reasonable estimate is that the errors introduced by the above mentioned effects would be about $7 \%$ in the $\mathrm{H}$ II regions, and about $3 \%$ in the $\mathrm{BK}$, for the composed $\mathrm{H} \alpha$ image. For the $\mathrm{H} \beta$ image, considering the increasing influence of the airmass with $\lambda^{-1}$, we obtain errors of $10 \%$ and $4 \%$, respectively.

\section{DISCUSSION \\ 4.1 Measured Fluxes}

In Table 3 we present the integrated absolute fluxes using aperture photometry of the $\mathrm{H}$ II regions identified in Fig. 1(a) by their emission peak, and whose center has been determined using the IRAF package. These values are corrected for internal reddening considering an intrinsic ratio of $\mathrm{H} \alpha /$ $\mathrm{H} \beta=2.88$. Lines and continua have been corrected as usual.

It can be seen from the reddening index $C(\mathrm{H} \beta)$ in Table 3 that regions 9 and 10 present a fairly large, $\mathrm{H} \alpha / \mathrm{H} \beta$ ratio, which can result from a larger influence of the underlying $\mathrm{H} \beta$ absorption than that adopted from MRS. In fact, MRS's Fig. 12(a) shows that the higher the $\log ([\mathrm{O} \mathrm{III}] / \mathrm{H} \beta)$, the larger the scattering around the mean equivalent width of the underlying $\mathrm{H} \beta$ absorption. Likewise, for the $\mathrm{BK}$, the average value of $C(\mathrm{H} \beta) \approx 0.85$ quoted is too high compared to that reported by Dinnerstein \& Shields (1986) of $C(\mathrm{H} \beta) \simeq 0.36$. This could be an effect of the strong underlying absorption (these authors assumed an absorption of $1 \AA \mathrm{EW}$ in Balmer lines).

The lines [O III] $\lambda 4959+5007 \AA$ are much more intense than $\mathrm{H} \beta \lambda 4861$. Figure 2 shows the behavior of $[\mathrm{O} \mathrm{III}] / \mathrm{H} \beta$ from each region as a function of its distance to the BK's center. There is a strong excitation gradient along the chain of $\mathrm{H}$ II regions.

In principle, it may be assumed that the excitation gradient corresponds to an abundance gradient (Zaritsky et al. 1989), and as in MRS's Fig. 12, we can obtain the metallicity gradient from the empirical relations [O III]/H $\beta$ and [O II $] /$ $\mathrm{H} \beta$ with $([\mathrm{O} \mathrm{III}]+[\mathrm{O} \mathrm{II}]) / \mathrm{H} \beta$, and estimating the fraction $[\mathrm{O}$ II $] / \mathrm{H} \beta$ (see Figs. 3 and 9 and Table 7 of MRS) using 
TABLE 3. Column 1: H II regions identification. Column 2 to 7 : lines flux in erg $\mathrm{cm}^{-2} \mathrm{~s}^{-1}$ and continuum intensities in erg cm $\mathrm{cm}^{-2} \mathrm{~s}^{-1}$; column 8: Absorption correction at $\mathrm{H} \beta$. Column 9: $\mathrm{H} \beta$ equivalent width. Column 10: $\mathrm{H} \beta$ luminosity in erg s $\mathrm{s}^{-1}$.

\begin{tabular}{ccccccccrc}
\hline \hline region & 4556 & 4861 & $4959+5007$ & 5290 & 6300 & 6563 & $\mathrm{C}(\mathrm{H} \beta)$ & $\mathrm{W}_{\boldsymbol{H} \beta}$ & $\log \mathrm{L}(\mathrm{H} \beta)$ \\
$\#$ & cont & $\mathrm{H} \boldsymbol{\beta}$ & {$[\mathrm{OIII}]$} & cont & cont & \multicolumn{1}{c}{$\boldsymbol{H} \boldsymbol{\alpha}$} & mag & $\AA$ & \\
\hline 1 & -15.13 & -13.81 & -13.67 & -15.17 & -15.26 & -13.70 & 0.00 & 21 & 38.75 \\
2 & -15.23 & -13.99 & -13.73 & -15.27 & -15.39 & -13.55 & 0.00 & 17 & 38.57 \\
3 & -15.44 & -14.50 & -13.81 & -15.50 & -15.70 & -14.04 & 1.05 & 9 & 38.06 \\
4 & -14.18 & -13.29 & -12.68 & -14.76 & -15.05 & -12.82 & 2.92 & - & - \\
5 & -15.64 & -14.63 & -14.54 & -15.68 & -15.84 & -14.38 & 0.00 & 10 & 37.93 \\
6 & -15.80 & -14.23 & -14.02 & -15.84 & -15.92 & -13.88 & 0.00 & 37 & 37.93 \\
7 & -15.05 & -13.70 & -13.14 & -15.09 & -15.32 & -13.24 & 0.55 & 23 & 38.86 \\
8 & -15.82 & -14.39 & -14.00 & -15.87 & -16.02 & -13.92 & 0.35 & 27 & 38.17 \\
9 & -14.01 & -13.39 & -12.50 & -14.12 & -14.86 & -12.93 & 5.10 & 4 & 39.17 \\
10 & -14.13 & -13.12 & -12.21 & -14.20 & -14.89 & -12.66 & 4.78 & 10 & 39.44 \\
11 & -15.36 & -14.01 & -13.64 & -15.41 & -15.62 & -13.55 & 1.23 & 22 & 38.55 \\
12 & -14.69 & -13.22 & -12.28 & -14.75 & -15.09 & -12.76 & 2.00 & 29 & 39.34 \\
13 & -15.62 & -13.36 & -12.57 & -15.80 & -16.02 & -12.90 & 1.73 & 185 & 39.20 \\
14 & -15.72 & -13.94 & -13.27 & -15.80 & -16.10 & -13.48 & 1.23 & 61 & 38.62 \\
15 & -15.50 & -13.75 & -13.01 & -15.57 & -15.84 & -13.28 & 1.35 & 57 & 38.81 \\
16 & -15.01 & -13.10 & -12.35 & -15.07 & -15.33 & -12.64 & 1.20 & 81 & 39.46 \\
17 & -15.67 & -13.90 & -13.35 & -15.76 & -15.99 & -13.43 & 0.80 & 58 & 38.66 \\
18 & -15.69 & -13.49 & -12.77 & -15.75 & -15.99 & -13.03 & 0.85 & 158 & 39.07 \\
19 & -15.50 & -13.50 & -12.74 & -15.54 & -15.84 & -13.04 & 0.88 & 99 & 39.06 \\
20 & -15.87 & -13.43 & -12.67 & -15.95 & -16.14 & -12.97 & 0.85 & 269 & 39.13 \\
21 & -15.12 & -13.18 & -12.39 & -15.18 & -15.57 & -12.71 & 1.30 & 86 & 39.38 \\
22 & -15.95 & -13.95 & -13.30 & -15.99 & -16.20 & -13.51 & 0.00 & 102 & 38.61 \\
23 & -15.80 & -13.94 & -13.28 & -15.84 & -16.02 & -13.51 & 0.00 & 71 & 38.62 \\
24 & -15.76 & -13.87 & -13.10 & -15.78 & -15.95 & -13.41 & 1.30 & 77 & 38.69 \\
25 & -15.00 & -13.10 & -12.35 & -15.04 & -15.36 & -12.64 & 0.70 & 79 & 39.46 \\
26 & -15.48 & -13.14 & -12.41 & -15.54 & -15.76 & -12.68 & 0.75 & 217 & 39.42 \\
27 & -15.53 & -13.33 & -12.74 & -15.60 & -15.80 & -12.86 & 0.78 & 159 & 39.22 \\
28 & -15.78 & -13.69 & -13.06 & -15.85 & -16.10 & -13.23 & 1.10 & 120 & 38.87 \\
\hline \hline & & & & & & & & & \\
& & & & & & & & \\
\hline
\end{tabular}

$$
\begin{aligned}
\log \left(\frac{[\mathrm{O} \text { II }]}{\mathrm{H} \beta}\right)= & 0.4371+0.248 X-0.3955 X^{2} \\
& -0.0766 X^{3}
\end{aligned}
$$

where

$$
X=\log \left(\frac{[\mathrm{O} \mathrm{III}]}{\mathrm{H} \beta}\right) .
$$

Thus, if $\log ([\mathrm{O} \mathrm{III}] / \mathrm{H} \beta)$ varies from 0 to 0.832 (see Fig. 2), $12+\log [\mathrm{O} / \mathrm{H}]$ could vary from 7.3 to 8.0 or from 8.7 to 8.0 , given the double-valued character of the relationship of $12+\log (\mathrm{O} / \mathrm{H})$ vs $([\mathrm{O} \mathrm{II}]+[\mathrm{O} \mathrm{III}]) / \mathrm{H} \beta$ (Edmunds \& Pagel 1984; Edmunds 1992). Nevertheless, the lower abundance range should be preferred in this case, since for $12+\log (\mathrm{O} /$ $\mathrm{H})=8.7$ the corresponding value $\log ([\mathrm{O} \mathrm{III}] /[\mathrm{N} \mathrm{II}])=0.1$ is too high when compared with the observations (Dinnerstein \& Shields 1986).

This fact is rather peculiar, because abundance gradients normally appear in the radial direction of the disks of spiral galaxies, which does not describe either the morphology of NGC 4861, or the distribution of its H II regions. If IC 3961 is the disk component associated with NGC 4861 (Dinnerstein \& Shields 1986), it is practically dust-free, when compared to spirals, and the $\mathrm{H}$ II regions would be distributed along its rim, because we cannot see sufficient dust absorption in the $\mathrm{H}$ II regions, as if they were distributed along spiral arms seen from the edge. They have to be external to the disk's dust. However, the implied gradient would not be typical of regions on a disk rim, i.e., where all of them are nearly at the same distance from the galactic center (the galaxy center taken as the center of the BK for reference, since it is the brightest point). The best morphological type for IC 3961 and NGC 4861 would be irregular, and it is well known that gas-rich disks and irregular galaxies do not present or, at most, have very low abundance gradients (Pagel et al. 1978).

It could happen, as in the case of M51, that the BK is a satellite of an older disk (IC 3961), and that the chain of H II regions constitutes a bridge linking both objects. An edge-on view of M51 would show a forearm linking the main galaxy to the satellite, showing certain aspects that resemble the chain of $\mathrm{H}$ II regions in NGC 4861. Nevertheless, an edge-on view of M51 would present evidence of dust, and its bulge would be visible, which is not the case of NGC 4861, whose structure is relatively amorphous. A more similar object is NGC 6412 (Arp 38), which presents a strongly emitting external arm, on the rim of the disk and terminating in a very bright object (similar to the BK) composed of a cluster of small $\mathrm{H}$ II regions. An edge-on view of Arp 38, from the 
(a)

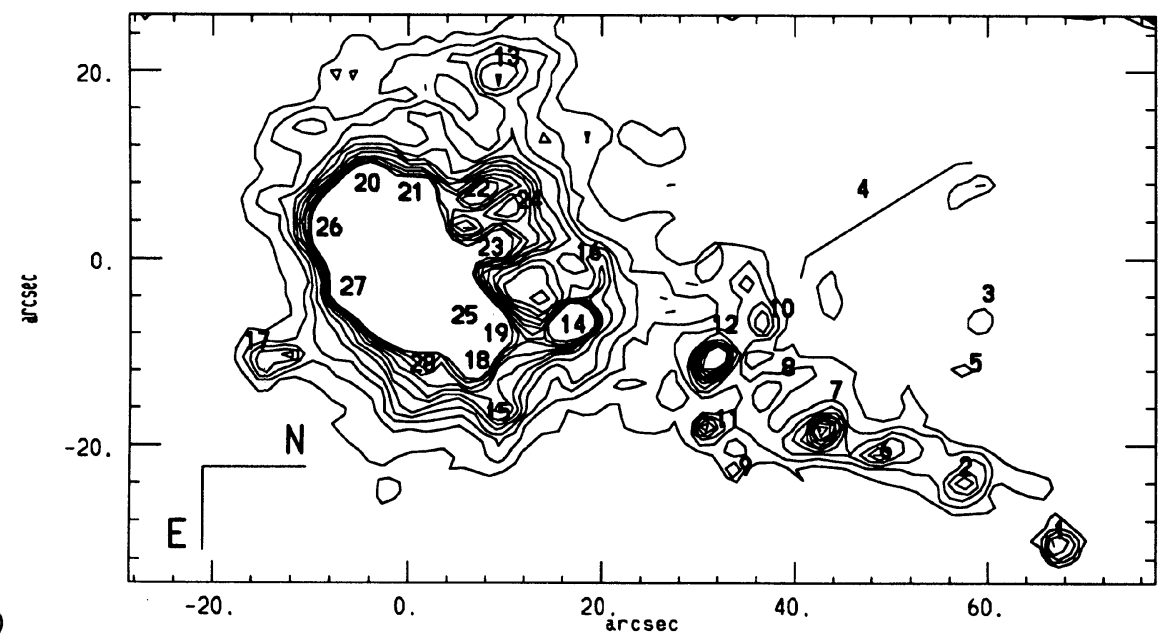

(b)
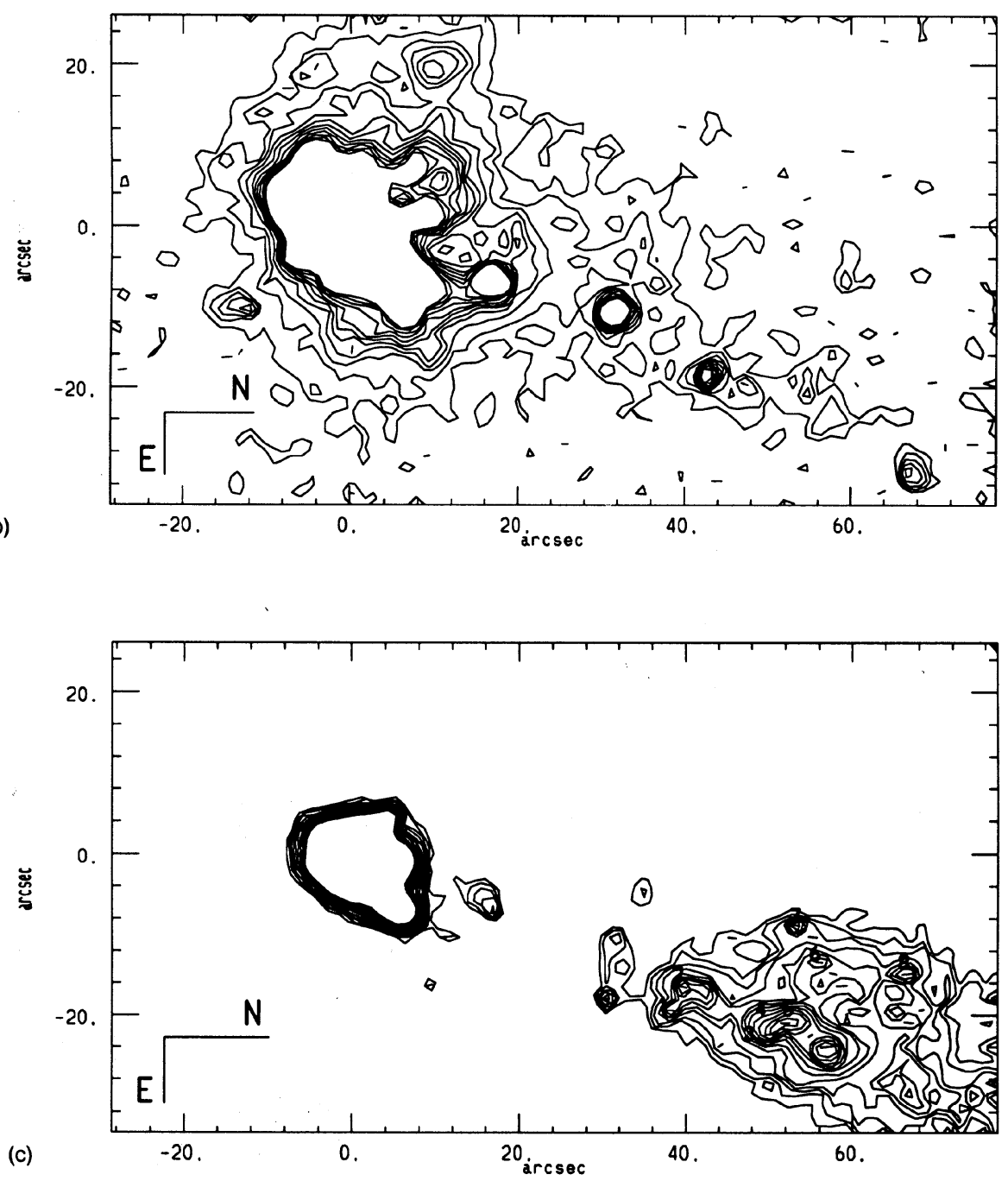

FIG. 1. (a) Isodensity map showing the $\mathrm{H}$ II regions studied in this work with their corresponding numbers. Scale and orientation is as shown. (b) Isodensity map of the [O III] emission. Scale and orientation as indicated. (c) Isodensity map showing the emissionless associations and the background companion IC 3961. Scale and orientation as indicated. 


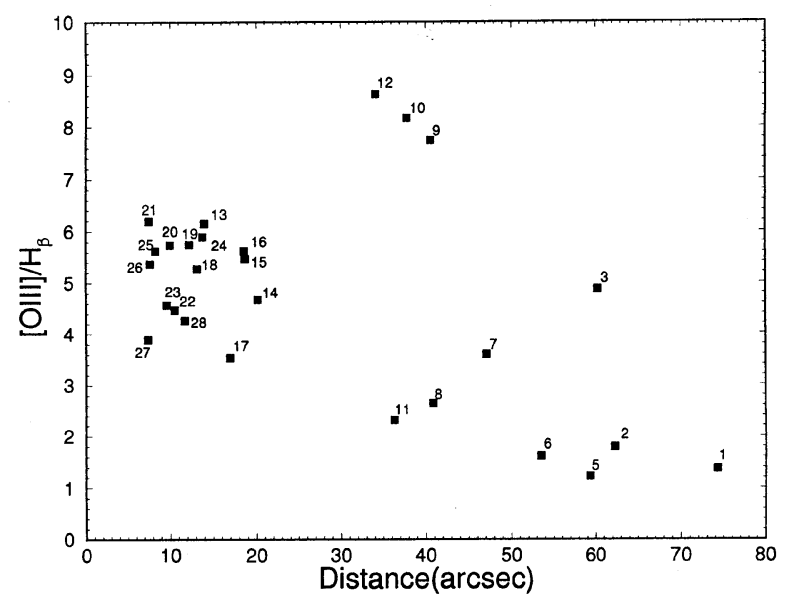

FIG. 2. Excitation index vs radial distance to the center of the BK, showing a gradient along the tail of $\mathrm{H}$ II regions.

northeast, would show an object similar to NGC 4861 (plus IC 3961, the disk component). The small tiny bulge of Arp 38 would be obscured by dust in the disk.

Another possible explanation might be that the excitation gradient is related to an evolutionary gradient. Copetti $e t$ al. (1986, hereafter referred to as CPD86) have shown that for a given metallicity, the index $\log ([\mathrm{O} \mathrm{III}] / \mathrm{H} \beta)$ evolves with the $\mathrm{H}$ II region's age, changing drastically in less than a million years. This interpretation seems more plausible, provided that the similar behavior of the equivalent width of $\mathrm{H} \beta$ $[W(\mathrm{H} \beta)]$ is taken into account, as explained in Sec. 4.3. However, this interpretation is unusual in the literature, where it is generally accepted that the excitation gradient is coupled to an abundance gradient in spirals (Vilchez \& Pagel 1988).

Regions 9, 10, and 12 present a very high excitation, $[\mathrm{O} \mathrm{III}] / \mathrm{H} \beta \simeq 8$. In Fig. 1 we see that these regions are distributed in an arc-like structure, centered in the emissionless association number 2 (see Table 5). This suggests a wavefront (probably originated in a supernova explosion), in which case the [O III] would be suffering extra ionization by shocks, shifting the mentioned regions to their present position in the diagram of Fig. 2.

The excitation index presents a correlation with the equivalent width of $\mathrm{H} \beta$ (Fig. 3), and also with the total flux of Lyman photons (Fig. 4), which is calculated from the intensity of $\mathrm{H} \beta$ :

$$
N_{912}^{0}=2.1 \times 10^{12} I_{\mathrm{H} \beta},
$$

where $N_{912}^{0}$ is the number of ionizing photons.

The intensity of the continuum does not present correlation with the excitation index. The stellar formation in the low-excitation regions $(1,2,5$, and 8$)$ is as intense as that in the high-excitation ones $(20,24,28$, etc. $)$. We see two possible explanations for this fact:

(a) The upper mass limit (UML) of the IMF is abundance-dependent, as described by Viallefond (1985). Then the less excited regions (more metallic) would present a lower UML.

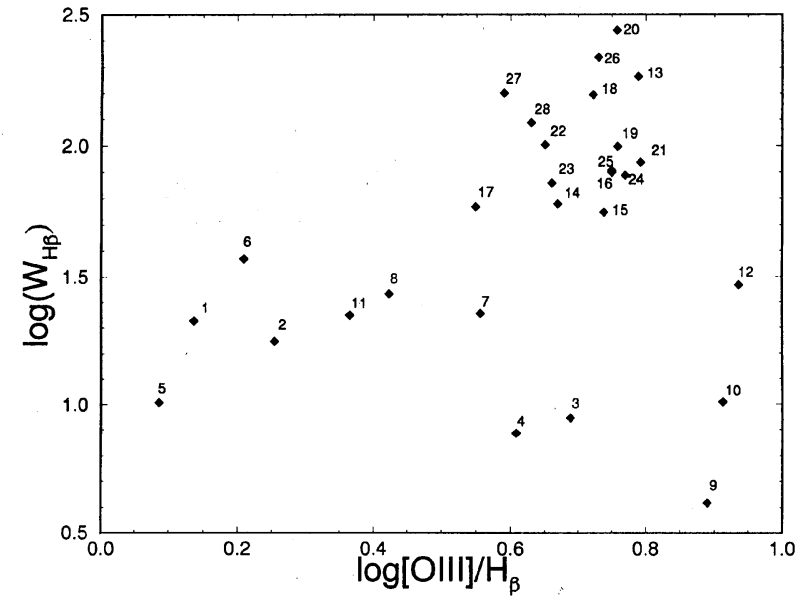

FIG. 3. Logarithm of $\mathrm{H}_{\beta}$ equivalent width against the excitation index.

(b) The $\mathrm{H}$ II regions are all of the same metallicity, but are in different states of evolution (CPD86). That is, the more massive stars have already left the MS in the older $\mathrm{H}$ II regions (the less excited ones).

We prefer the last hypothesis since, as we will discuss in Sec. 4.3, $W(\mathrm{H} \beta)$, colors and excitation all correlate with distance from the BK.

\subsection{The $B K$}

The BK's integrated fluxes are given in Table 4 for lines and continua measured with different diaphragms. By comparing the $\mathrm{H} \beta$ flux measured through a 5 pixels diaphragm $\left(\approx 2^{\prime \prime}\right)$ with that of the $\mathrm{H}$ II regions measured with the same diaphragm, we infer that the BK is one order of magnitude more intense than the strongest regions $(10,16$, and 25$)$. The BK $\mathrm{H} \beta$ luminosity is 5 times larger than that of the "Jumbo" region in NGC $3310\left[L(\mathrm{H} \beta)=4.4 \times 10^{39} \mathrm{erg} \mathrm{s}^{-1}\right.$; Pastoriza et al. 1992], and 6 times larger than that of 30 Doradus.

The $\mathrm{H} \beta$ continuum of the $\mathrm{BK}$ is $10-30$ times more intense than in the other regions, indicating that a remarkable

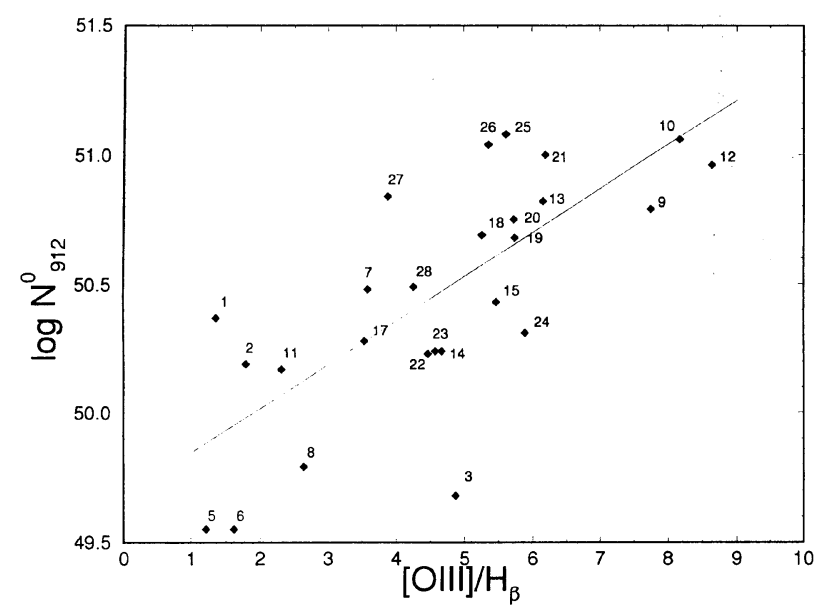

FIG. 4. Logarithm of the total flux of Lyman photons against the excitation index. 
TABLE 4. BK integrated fluxes through successive diaphragms. Radii are in pixels and the scale is 1 pix $=0.303$ arcsec. Lines flux in erg $\mathrm{cm}^{-2} \mathrm{~s}^{-1}, \mathrm{continuum}$ intensities in erg cm ${ }^{-2} \mathrm{~s}^{-1} \AA^{-1}$ and $\mathrm{H} \beta$ luminosity in erg s${ }^{-1}$.

\begin{tabular}{cccccccccc}
\hline \hline $\begin{array}{c}\text { radius } \\
\text { pixel }\end{array}$ & $\begin{array}{c}4556 \\
\text { cont }\end{array}$ & $\begin{array}{c}4861 \\
\mathbf{H} \beta\end{array}$ & $\begin{array}{c}4959+5007 \\
{[\mathrm{OIII}]}\end{array}$ & $\begin{array}{c}5290 \\
\text { cont }\end{array}$ & $\begin{array}{c}6300 \\
\text { cont }\end{array}$ & $\begin{array}{c}6563 \\
\mathrm{H} \boldsymbol{\alpha}\end{array}$ & $\begin{array}{c}\mathrm{C}(\mathrm{H} \beta) \\
\mathrm{mag}\end{array}$ & $\begin{array}{c}\mathrm{W}_{H \beta} \\
\AA\end{array}$ & $\log \mathrm{L}(\mathrm{H} \beta)$ \\
\hline 5 & -14.20 & -12.08 & -11.17 & -14.26 & -14.62 & -11.62 & 1.15 & 133 & 40.48 \\
10 & -13.94 & -11.76 & -10.89 & -13.98 & -14.31 & -11.30 & 0.98 & 150 & 40.80 \\
15 & -13.88 & -11.69 & -10.85 & -13.92 & -14.21 & -11.23 & 0.88 & 153 & 40.87 \\
20 & -13.83 & -11.66 & -10.83 & -13.87 & -14.17 & -11.19 & 0.85 & 147 & 40.90 \\
25 & -13.77 & -11.63 & -10.81 & -13.82 & -14.11 & -11.16 & 0.85 & 138 & 40.93 \\
30 & -13.73 & -11.60 & -10.78 & -13.78 & -14.06 & -11.13 & 0.85 & 134 & 40.96 \\
35 & -13.71 & -11.58 & -10.77 & -13.76 & -14.03 & -11.11 & 0.85 & 134 & 40.98 \\
40 & -13.70 & -11.57 & -10.76 & -13.74 & -14.02 & -11.10 & 0.85 & 134 & 40.99 \\
45 & -13.67 & -11.56 & -10.75 & -13.73 & -14.01 & -11.09 & 0.85 & 131 & 41.00 \\
65 & -13.59 & -11.50 & -10.71 & -13.65 & -13.93 & -11.04 & 0.85 & 127 & 41.06 \\
\hline \hline
\end{tabular}

burst of star formation occurred therein. The intensity of [O III] $\lambda 5007$ (see Table 4) decreases drastically outwards in the BK, suggesting that the source of excitation also presents a strong degree of concentration.

Assuming that the 65 pixels diaphragm includes the whole BK, we can estimate additional parameters of its stellar population. By comparing $(\mathrm{H} \beta)$ with $\mathrm{CPD} 86$ evolution curves, approximating the $\mathrm{BK}$ metallicity by $\simeq 10 \%$ solar, and assuming a Salpeter IMF $(\chi=1.35)$, with an upper mass limit of $70 \mathscr{L}_{\odot}$, we obtain an upper limit for the $\mathrm{H}$ II region's age of $4.5 \times 10^{6} \mathrm{yr}$, considering a model without mass loss.

Cid-Fernandes et al.'s (1992) models of stellar population synthesis allow us to estimate the mass of stars formed within the BK: for $1.35 \leqslant \chi \leqslant 2.0$ the range of total masses of the ionizing cluster are $2.2 \times 10^{6} \mathscr{M}_{\odot} \leqslant \mathscr{M}_{T} \leqslant 6.7 \times 10^{6} \mathscr{M}_{\odot}$ and, $1.6 \times 10^{4} \mathscr{C b}_{\odot} \leqslant \mathscr{M b}_{I} \leqslant 2.2 \times 10^{4} \mathscr{M b}_{\odot}$ for the ionizing stars.

The BK brightness distribution as derived from the three lines and three continua is $-2.04 \pm 0.35$ (Dottori et al. 1993).

\subsection{The Colors of the Emissionless Regions and IC 3961}

In Table 5 we present the integrated flux of the continua for eight emissionless associations, clearly detected in the three continuum frames, and numbered according to Fig. 1(c). The second region is the center of the arcs formed by the $\mathrm{H}$ II regions $8,9,10,11$, and 12 (and probably region 7 ), as previously mentioned. Their continuum slope is less steep than that of the ionizing associations.

In Fig. 5 we show the dependence of the equivalent width

TABLE 5. Logarithm of continuum intensities (in erg $\mathrm{cm}^{-2} \mathrm{~s}^{-1} \AA^{-1}$ ) at the filters center in the emissionless associations.

\begin{tabular}{ccccc}
\hline $\begin{array}{c}\text { region } \\
\#\end{array}$ & $\begin{array}{c}\text { radius } \\
\text { pixel }\end{array}$ & $\begin{array}{c}4556 \\
(\mathrm{H} \beta)_{\mathrm{C}}\end{array}$ & $\begin{array}{c}5290 \\
{[\mathrm{OIII}]_{\mathrm{C}}}\end{array}$ & $\begin{array}{c}6300 \\
(\mathrm{H} \alpha)_{\mathrm{C}}\end{array}$ \\
\hline 1 & 5 & -16.13 & -16.27 & -16.39 \\
2 & 5 & -16.06 & -16.16 & -16.34 \\
3 & 3 & -16.52 & -16.69 & -16.71 \\
4 & 4 & -16.24 & -16.34 & -16.45 \\
5 & 3 & -16.52 & -16.64 & -16.80 \\
6 & 5 & -16.16 & -16.28 & -16.40 \\
7 & 5 & -16.15 & -16.32 & -16.41 \\
8 & 5 & -16.17 & -16.28 & -16.37 \\
\hline \hline
\end{tabular}

of $\mathrm{H} \beta[W(\mathrm{H} \beta)]$ on the color $5290-6300$, obtained as the difference between the values quoted in Table 3 for the $\mathrm{H}$ II knots. It is clear from the figure that the colors of knots 9 and 10 appear to be different than those of the rest of the knots, reinforcing the suggestion of an additional excitation mechanism. On the other hand, the tail's H II knots present systematically lower $W(\mathrm{H} \beta)$ values and lower values of the 5290 -6300 index than the ones around the BK. This effect is missing in the corresponding plot including the color index 4556-5290. In Fig. 6, we show the composite color-color diagram constructed using both indices 4556-5290 and 5290-6300; the diagram also includes the emissionless associations. H II regions 9,10 , and 4 exhibit striking colors; regions 9 and 10, possibly as a consequence of a shock excitation, show an enhanced "green" 5290-6300 index with respect to the whole sample of $\mathrm{H}$ II regions. By contrast, knot 4 presents an extremely blue color which, by inspection of Figs. 1(a) and 1(b), is probably affected strongly by measurement errors due to its extended nature and faintness. Apart from these cases, this diagram differentiates clearly emissionless knots (open squares) from $\mathrm{H}$ II regions 1 to 8 (NE part of the tail, black circles in Fig. 6), and 13 to 28 (that

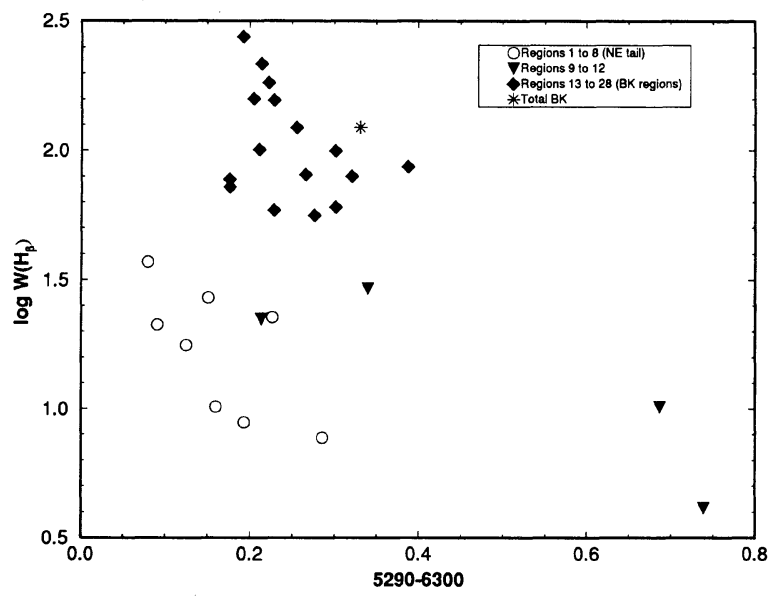

FIG. 5. Logarithm of the $\mathrm{H} \beta$ equivalent width $[W(\mathrm{H} \beta)]$ vs color 5290 -6300 . Different symbols denote different knots, as indicated. Colors of knots 9 through 12 appear to be different than the rest of the knots. 


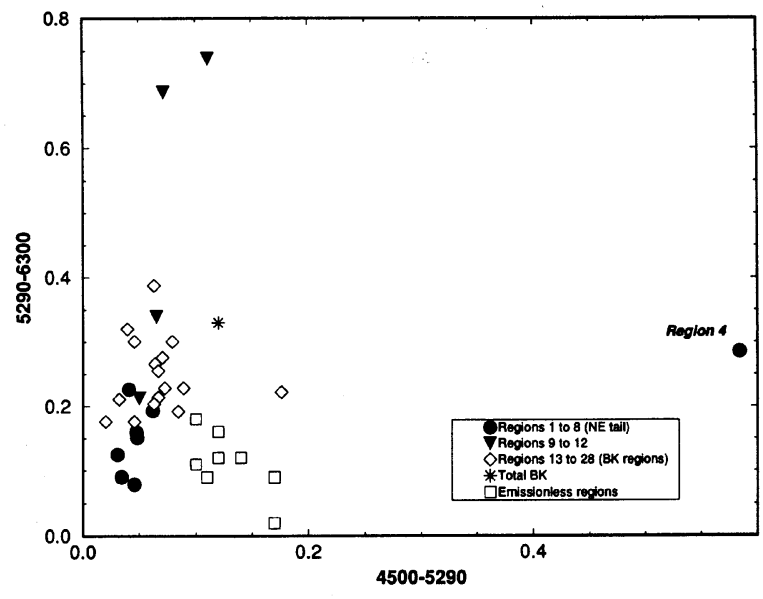

Fig. 6. Color 5290-6300 vs color 4556-5290. Different symbols denote different knots, as indicated. Knots 4,9 , and 10 present very striking colors.

surrounding the BK, open diamonds in Fig. 6), the latter ones presenting smaller 4556-5290 colors and extending the values of the 5290-6300 index to higher values than the emissionless knots. All these facts point to a gradient in the star formation activity and the evolutionary status going from the BK towards the North, along the tail and the underlying companion. The gradients in the excitation and $W(\mathrm{H} \beta)$, are accompanied by a clear gradient in the color 5290-6300, as we can see in Fig. 7. This gradient is not present in the color 4556-5290.

This change in the evolutionary status along the tail suggests the possibility that a self-propagated star formation process (Seiden \& Gerola 1982), originated in or near knot number 1 , has propagated along the tail towards the $\mathrm{BK}$, which itself seems to be the site of the most recent star formation.

The age of the $\mathrm{H}$ II regions depends upon the model employed. They have been evaluated both using CPD86 and Mas-Hesse \& Kunth's (1991) models. The lowest velocity

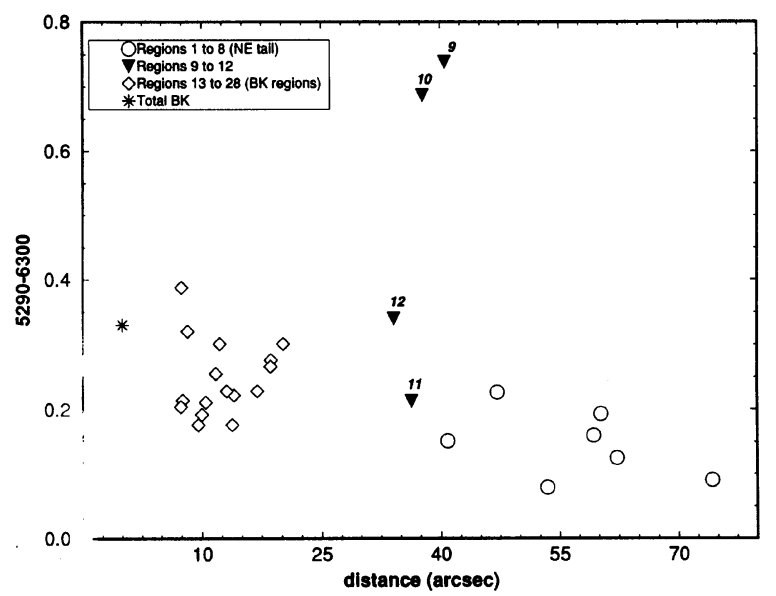

FIG. 7. Color 5290-6300 as a function of the distance to the BK. Different symbols denote different knots, as indicated. A color gradient which correlates with the excitation gradient can be clearly seen. inferred from those ages for the propagation of a star formation front is some $400 \mathrm{~km} / \mathrm{s}$. Seiden \& Gerola (1982) claim that stochastic star formation is more likely to be due to supernova explosions. This scenario is compatible with the rate of 0.01 supernova $\mathrm{yr}^{-1}$ found by Sramek \& Weedman (1986).

The continuum colors of IC 3961 have been measured west and southward of the chain of the $H$ II regions. The flux ratios are:

$$
\begin{aligned}
& \log \frac{[\mathrm{O} \mathrm{III}] c}{\mathrm{H} \alpha c}=0.08, \\
& \log \frac{\mathrm{H} \beta c}{[\mathrm{O} \mathrm{III}] c}=0.10 .
\end{aligned}
$$

Comparing these values with the tables of O'Connell (1973), we see that the stellar population might be of type F0-5, blue but not ionizing, which reinforces the fact that no evidence was found of emission lines in IC 3961. No stellar formation has occurred in IC 3961 at least in the last $3 \times 10^{7}$ yr.

\section{CONCLUSIONS}

In this work we have analyzed the galaxy NGC 4861 through images taken with narrowband interference filters on the lines $\mathrm{H} \alpha, \mathrm{H} \beta$, and [O III] $\lambda 5007$ and nearby continua calibrated absolutely through spectrophotometric standard stars.

The galaxy NGC 4861 presents strong [O III] emission. Analysis of $28 \mathrm{H}$ II regions shows the existence of an excitation gradient along the chain of $\mathbf{H}$ II regions. This phenomenon can be due to an abundance gradient or to a difference in the evolutionary stage (or age) of the $\mathrm{H}$ II regions. The last possibility is reinforced by the fact that stellar formation in the low excitation regions is as strong as that in the high excitation regions. The excitation, the color 5290-6300 and the $W(\mathrm{H} \beta)$ gradients, suggest that star formation has propagated along the tail of $\mathrm{H}$ II regions towards the BK, at supersonic velocities of order several hundreds of $\mathrm{km} \mathrm{s}^{-1}$. The origin of this star formation can be located in the knots closer to IC 3961, and probably channeled via the geometry of the gas distribution of NGC 4861 .

In the $\mathrm{BK}$ we see that the strong [O III] emission is more intense and concentrated than $\mathrm{H} \alpha$ emission, indicating low metallicity and a high concentration of the burst of star formation in the center of the structure.

We do not detect emission lines in the companion galaxy IC 3961. Its continuum colors correspond to a blue population predominantly of type F0-F5, indicating that the last strong cycle of stellar formation is as old as $30 \mathrm{Myr}$.

In the BK we measured a very intense continuum, stronger than that of the other $\mathrm{H}$ II regions, indicating a huge burst of star formation. Although many "Strömgren spheres" are observed all around the BK, the strongest activity of star formation occurred at its very center.

For the last cycle of star formation in the BK we obtained an age of $4.5 \mathrm{Myr}$, and a mass of $2.21 \times 10^{6} \mathscr{M}_{\odot}$ for a Sal- 
peter IMF, the mass of ionizing stars being $1.55 \times 10^{4} \mathscr{M b}_{\odot}$. The continuum of the eight emissionless associations is flatter than that of the $\mathrm{H}$ II regions.

The Jacobus Kapteyn Telescope is operated on the island of La Palma by the Royal Greenwich Observatory in the Spanish Observatorio del Roque de Los Muchachos of the Instituto de Astrofísica de Canarias. This research has made use of the NASA/IPAC extragalactic database (NED) which is operated by the Jet Propulsion Laboratory, California Institute of Technology, under contract with the National Aeronautics and Space Administration. This work was partially financed by the Brazilian institution CNPq and FINEP. JC and JMV acknowledge partial support through Grants PB89 -0510 and PB91-0525 from the Dirección General de Investigación Científica y Técnica of the Spanish Ministerio de Educación y Ciencia.

\section{REFERENCES}

Arp, H. 1966, ApJS, 14, 1

Christian, C. A., Adams, M., Barnes, J. V., Butcher, H., Hayes, D. S., Mould, J. R., \& Siegel, M. 1985, PASP, 97, 363

Cid-Fernandes, R., Dottori, H., Viegas, S., \& Gruenwald, R. 1992, MNRAS, 255,165

Copetti, M. V. F., Pastoriza, M. G., \& Dottori, H. 1986, A\&A, 156, 111 (CPD86)

de Vaucouleurs, G., de Vaucouleurs, A., \& Corwin, H. G. 1976, Second Reference Catalogue of Bright Galaxies (University of Texas)

Dinnerstein, H. L., \& Shields, G. A. 1986, ApJ, 311, 45

Dottori, H., Cepa, J., Vilchez, J., \& Barth, C. S. 1994, A\&A, 283, 753

Edmunds, M. G., \& Pagel, B. E. J. 1984, MNRAS, 211, 507

Edmunds, M. G. 1992, in Elements and the Cosmos, edited by M. G. Edmunds and R. J. Terlevich (Cambridge University Press, Cambridge), p. 289

Fisher, J. R., \& Tully, R. B. 1981, ApJS, 47, 139

Hodge, P. 1983, AJ, 3, 296

Mas-Hesse, J. M., \& Kunth, D. 1991, A\&AS, 88, 399
McCall, M. L., Rybski, P. M., \& Shields, G. A. 1985, ApJS, 57, 1 (MRS) O'Connell, R. W. 1973, AJ, 78, 1074

Oke, J. B., \& Gunn, J. 1983, ApJ, 266, 713

Pagel, B. E. J., Edmunds, M. G., Blackwell, D. E., Chun, M. S., \& Smith, G. 1979, MNRAS, 189, 95

Pagel, B. E. J., Edmunds, M. G., Fosbury, R. A. E., \& Webster, B. L. 1978, MNRAS, 184, 569

Pastoriza, M. G., Dottori, H., Terlevich, E., Terlevich, R., \& Diaz, A. 1992, MNRAS (in press)

Searle, L. 1971, ApJ, 168, 327

Seiden, P. E., \& Gerola, H. 1982, Fund. Cosmic Phys., 7, 241

Sramek, R. A., \& Weedman, D. W. 1986, ApJ, 302, 640

Viallefond, F. 1985, in Star-forming Dwarf Galaxies and Related Objects, edited by Ed. D. Kunth, T. X. Thuan, and J. Tran Thanh Van, p. 207

Vilchez, J. M., \& Pagel, B. E. J. 1988, MNRAS, 231, 257

Zaritsky, D., Elston, R., \& Hill, J. M. 1989, AJ, 97, 97

Zwicky, F. 1961, Catalogue of Galaxies and Clusters of Galaxies (California Institute of Technology, Pasadena) 